\title{
Apparent Scarcity of Low-Density Polymorphs of Inorganic Solids
}

\author{
Martijn A. Zwijnenburg, ${ }^{1}$ Francesc Illas, ${ }^{1}$ and Stefan T. Bromley ${ }^{1,2, *}$ \\ ${ }^{1}$ Departament de Química Física and Institut de Química Teòrica i Computacional, Universitat de Barcelona, \\ E-08028 Barcelona, Spain \\ ${ }^{2}$ Institució Catalana de Recerca i Estudis Avançats (ICREA), E-08010 Barcelona, Spain
}

(Received 8 January 2010; published 30 April 2010)

\begin{abstract}
For most inorganic solids, very few dense polymorphs and no low-density polymorphs are observed. Taking a wide range of tetrahedrally-coordinated binary solids (e.g., $\mathrm{ZnO}, \mathrm{GaN}$ ) as a prototypical system, we show that the apparent scarcity of low- density polymorphs is not due to significant structural or energetic limitations. Using databases of periodic networks as sources of novel crystal structures, followed by $a b$ initio energy minimization, we predict a dense spectrum of low-density low-energy polymorphs. The diverse range of materials considered indicates that this is likely to be a general phenomenon.
\end{abstract}

PACS numbers: 61.50.Ah, 61.66.Fn, 71.15.Nc, 82.75.Fq

The structure and properties of inorganic solids are intimately linked to such an extent that even different structures of the same composition (so-called polymorphs) often have widely differing properties and applications. For boron nitride (BN), for example, the soft hexagonal layered polymorph $(h-\mathrm{BN})$ is used as a lubricant, while the cubic polymorph (wurtzite structure) is extremely hard and is employed as an industrial abrasive. Germanium also exhibits more than one polymorph (or more strictly "allotrope" for elemental systems) having very different optical properties; with the diamond structured $\alpha$-germanium having an indirect band gap, and the recently synthesized lowdensity clathrate-II structure [1] a direct gap. Evidently, having access to more than one polymorph can significantly extend the range of properties and applications of a particular compound. For the vast majority of inorganic solids, however, typically only three or fewer distinct polymorphs have been prepared experimentally and a similarly small number of hypothetical polymorphs have been proposed. Moreover, where more than one polymorph is known, these extra phases very rarely have a significantly lower density than the most stable polymorph.

In stark contrast to this situation, silica $\left(\mathrm{SiO}_{2}\right)$ has been prepared as more than 40 polymorphs [2], the majority of which are considerably less dense than $\alpha$-quartz; the most stable polymorph under ambient conditions. The rich polymorphism of silica, especially in its low-density forms, allows for fine-tuning of applications (e.g., gas separation membranes) by choosing the best suited polymorph. In addition, $100000+$ hypothetical silica polymorphs have been predicted theoretically as fourfold-connected networks (4CNs) [3-7], a large fraction of which, after accurate theoretical evaluation as silica materials, were found to have comparable energetics to experimentally prepared polymorphs [8,9]. Similarly rich polymorphism has only been further observed for a select group of other inorganic solids, for example, aluminophosphates $\left(\mathrm{AlPO}_{4}\right)$. A tantalizing hint that there may as yet exist a greater pool of experimentally accessible polymorphs for many other inorganic solids is given by the recent low temperature deposition synthesis of the tetrahedral wurtzite polymorph of $\mathrm{LiBr}$ [10] (after previous theoretical prediction [11]). While the wurtzite structure is still generally considered as dense, it is significantly less dense than the closed-packed rocksalt structure normally observed for $\mathrm{LiBr}$. Largely inspired by these studies we have used accurate density functional (DF) calculations to explore the energy landscape of a range of other binary solids (i.e., with atomic composition $A B$ ) with a view to establishing the prevalence of feasible low-density polymorphs. For all systems we predict a multitude of structures, many having remarkably open frameworks, lying surprisingly low in energy, thereby demonstrating that the apparent scarcity of low-density polymorphs is not due to significant structural and/or energetic limitations.

In our investigation we concentrate on a wide selection of the extensive class of 1:1 binary solids that crystallize experimentally at ambient conditions as tetrahedral polymorphs, reminiscent of those of silica. Just as for the silicon atoms in silica polymorphs, the atoms of these tetrahedrally-coordinated binary solids (hereafter referred to as TBSs) can be abstractly regarded as vertices of $4 \mathrm{CNs}$. TBSs have been generally prepared in only two tetrahedral polymorphs (wurtzite and zincblende), a number of intergrowths in between these two (so-called polytypes [12]) and at high pressure as the octahedral rocksalt polymorph $[13,14]$. Only one further polymorph is observed for TBSs experimentally: the $\beta$ - $\mathrm{BeO}$ or $\mathrm{BCT}$ structure (where the three letter code, here and throughout, refers to an International Zeolite Association framework type [15]), as displayed by $\mathrm{BeO}$ (i.e., $\mathrm{BCT}-\mathrm{BeO}$ ) at high temperatures [16], sharing the same $4 \mathrm{CN}$ as the silicate Mg-BCTT .

Previous theoretical work addressing the energy landscape of TBSs has identified a number of hypothetical polymorphs, many of which lie much higher in energy than wurtzite and zincblende. The two lowest energy 
hypothetical TBS polymorphs predicted to date are (i) $\mathrm{BCT}$, although known experimentally for $\mathrm{BeO}$, has also been proposed as a possible extended phase in $\mathrm{ZnO}$ and $\mathrm{ZnS}$ nanostructures (BCT-ZnO [17,18], BCT-ZnS [19]) and, (ii) the SOD polymorph (sharing the same underlying $4 \mathrm{CN}$ as the low-density silicate sodalite [15]) recently proposed for $\mathrm{ZnO}$ (SOD-ZnO [20]) and $\mathrm{SiC}$ (SOD-SiC [21]). Furthermore, for BN, which is not strictly a TBS as it crystallizes under ambient conditions as the three-connected $h$-BN polymorph, ABW-BN (again with a $4 \mathrm{CN}$ originally found as a silicate) and BCT-BN have been predicted [22]. Such studies have not exploited the huge resource of enumerated $4 \mathrm{CNs}$, but have instead derived TBS polymorphs based on methods inspired by physical processes (e.g., simulated thermal [22] and mechanical annealing [17], cluster assembly [20,21]). Arguably, the most thorough study of the energy landscape of any TBSlike system to date was that recently applied to BN [22] which sampled systems with up to only eight atoms per unit cell finding only two hypothetical TBS polymorphs (see above). Evidently, to date, such studies have yielded only a very small number of hypothetical TBS polymorphs with respect to those derived for silica from the enumeration of 4CNs [3-7]. Following this latter topological approach we here sample directly the large and diverse set of $4 \mathrm{CNs}$ (with up to 48 atoms per unit cell when realized as a TBS) from a variety of sources.

We initially started our exploration with 4CNs realized as $\mathrm{ZnO}$ materials. Taking a representative sample of 55 $4 \mathrm{CNs}$, all of which only contained even rings (the atomic ordering of TBSs does not allow for 4CNs with odd rings), we then constructed them as appropriately scaled $\mathrm{ZnO}$ structures. In a screening step, we next energy minimized the structures of all resulting $\mathrm{ZnO}$ materials using the GULP [23] code and the empirical potential of Whitmore et al. [24], which were then checked to be true energy minima by phonon calculations. Subsequently, we selected the 26 structures with the lowest energy, some of which are shown in Fig. 1. This selection was then reoptimized using periodic DF calculations for $\mathrm{ZnO}$ and a range of other TBSs: those from groups I-VII (AgI), II-VI ( $\mathrm{ZnS}, \mathrm{CdS})$, III-V $(\mathrm{GaP}, \mathrm{GaN})$ and $\mathrm{IV}(\mathrm{SiC})$. The initial structures for the rest of the materials considered were obtained by rescaling the optimized $\mathrm{ZnO}$ structures to obtain suitable bond lengths. In all DF calculations a combination of the gradient corrected PW91 [25] functional and projector augmented waves [26], as implemented in the VASP [27] code, were employed. To minimize spurious dependency of basis-set quality on unit-cell size, all reported energies and structures are after two sequential optimizations of both atomic positions and cell parameters and a final single point calculation. Calculations employed a $550 \mathrm{eV}$ plane-wave cutoff, with $k$-point grids chosen such that the energy differences between different polymorphs were converged to within $1 \mathrm{~kJ} / \mathrm{mol}$.

Figure 2 shows the resulting relative energies of the different polymorphs for the different TBSs plotted against
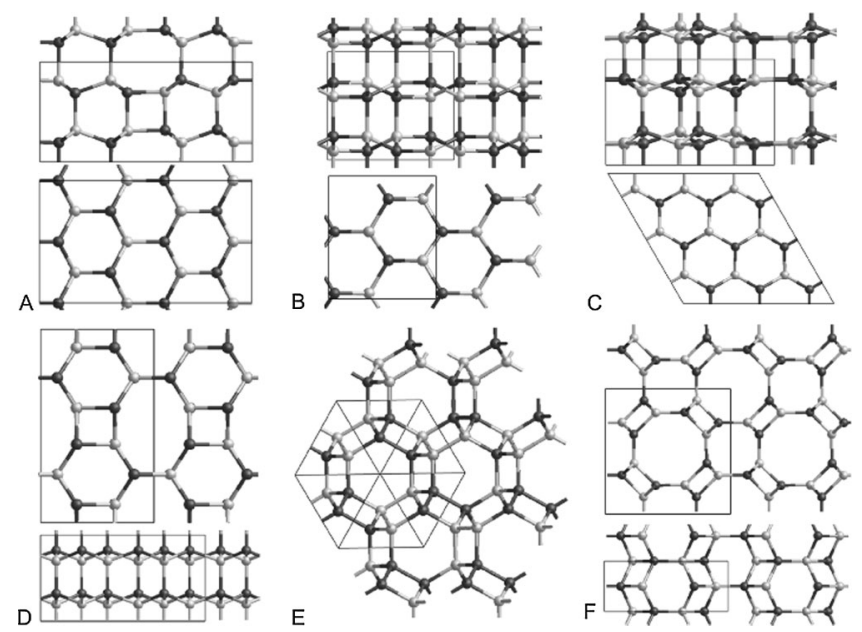

FIG. 1. Structures of selected low-energy hypothetical TBSs: (a) 59_4_280312 (3 kJ/mol), (b) 64_1_1 $(5 \mathrm{~kJ} / \mathrm{mol})$, (c) 189_3_1615 (8 kJ/mol), (d) ATV (13 kJ/mol), (e) SOD (13 kJ/mol), (f) ATN (17 kJ/mol). (a), (b), and (c) are based upon 4CNs from the database of Treacy et al. [1,4,38] and (d), (e), and (f) are derived from 4CNs known for porous crystalline silicates (i.e., zeolites) [15]. Energies in parentheses correspond to the energy difference of the $\mathrm{ZnO}$ form of the polymorph with respect to wurtzite- $\mathrm{ZnO}$. The unit cell is boxed in each case.

their relative molecular volume $\left(V_{m} / V_{m}\right.$ wurtzite $)$ and allows for three clear observations. First on a methodological note, in all cases the wurtzite and zincblende polymorphs and the $4 H$ and $6 H$ polytypes lie lowest in energy and their order of stability follows that found by calorimetry [28,29] and, where experimental data is currently unavailable, by previous theoretical work [30-32]. Second, for all TBSs studied we find a dense spectrum of polymorphs that are approximately evenly spaced over a wide range of relative molecular volumes, reaching up to relative molecular volumes at least $20 \%$ larger than that of wurtzite. When ordered with respect to energy, the average energy gap between each subsequent pair of polymorphs ranges from $0.4 \mathrm{~kJ} / \mathrm{mol} A B$ for $\mathrm{AgI}$ to $2 \mathrm{~kJ} / \mathrm{mol} A B$ for $\mathrm{SiC}$. As this measure is likely to decrease even further when considering more than 26 polymorphs, it provides an effective upper boundary to the coarseness of the polymorph spectrum. This suggests that the dense spectrum of low-density polymorphs observed for silica is not an exceptional property but general to all tetrahedrally-coordinated solids, and perhaps, more speculatively, all simple solids. Third, both the overall energetic ordering of polymorphs and the shapes of the curves for the different TBSs in Fig. 2 are very similar. This suggests that, even though the various TBSs considered contain different combinations of elements, the underlying physical relationship between topology and energy is strikingly similar.

The energy range spanned by the 26 polymorphs differs strongly between the different TBSs, from $0-9 \mathrm{~kJ} / \mathrm{mol} A B$ for $\mathrm{AgI}$ to $0-45 \mathrm{~kJ} / \mathrm{mol} A B$ for $\mathrm{SiC}$ and as shown in Fig. 3 appears to increase with the formal charges of the constituent ions. A significant component of the energy of TBS 


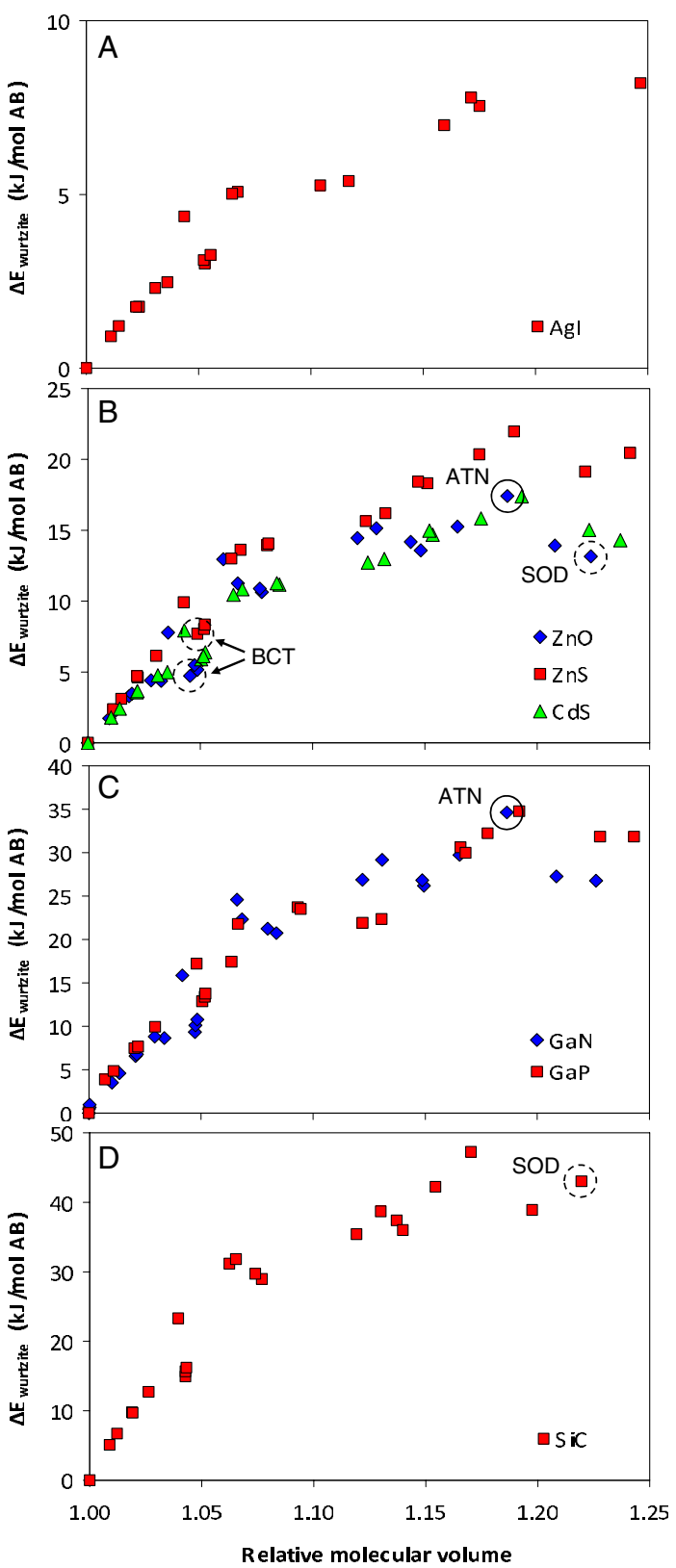

FIG. 2 (color online). Energies with respect to the wurtzite structure $\left(\Delta E_{\text {wurtzite }}\right)$ of a range of TBSs based on monovalent ions (a), divalent ions (b), trivalent ions (c), and tetravalent ions (d), plotted against their relative molecular volume $\left(V_{m} / V_{m \text { wurtzite }}\right.$ ). Points corresponding to previously predicted TBSs (Refs. [17-21]) are circled by dashed lines, while doped experimental TBSs are circled in black (Refs. [36,37]).

polymorphs and its link with topology is thus most likely electrostatic in nature. To put the above energy ranges in perspective, the rocksalt polymorph, that has been experimentally prepared and found to be metastable at atmospheric pressure for three TBSs ( $\mathrm{ZnO}$ [33], CdS [34] and $\mathrm{GaN}$ [35]), is calculated for all TBSs to lie significantly higher in energy than all 26 polymorphs considered (e.g., $+29 \mathrm{~kJ} / \mathrm{mol} A B$ for $\mathrm{ZnO},+25 \mathrm{~kJ} / \mathrm{mol} A B$ for $\mathrm{CdS}$, and $+94 \mathrm{~kJ} / \mathrm{mol} A B$ for $\mathrm{GaN}$ ).
The advantage of exploring the energy landscape of well-defined crystalline solids by first identifying likely minima via a topological approach can be seen by considering the number of new hypothetical low-energy, lowdensity TBS polymorphs found herein with respect to those previously predicted (i.e., $\mathrm{ABW}, \mathrm{BCT}$ and $\mathrm{SOD}$, see above). For all compositions studied over $90 \%$ of our 19 newly proposed hypothetical TBS polymorphs are found to lie lower in energy than SOD and ABW, while 20\%-25\% lie energetically below BCT. Other methods of searching for new TBS polymorphs which physically sample the energy landscape more directly may struggle to find these new polymorphs due to the combination of high structural diversity and the wide range of atoms per unit cell (4-48) and unit-cell volumes (e.g., $\sim 40-80 \AA^{3}$ for $\mathrm{ZnO}$ ) covered.

As a further note, it is tantalizing to observe that one low-density polymorph in our set of hypothetical polymorphs [ATN, see Fig. 1(f)] has been synthesized twice as a doped TBS: for $\mathrm{ZnO}$ as $|\mathrm{K}, \mathrm{Rb}|\left[\mathrm{Zn}_{3} \mathrm{LiO}_{4}\right]$ [36] and for $\mathrm{GaN}$ as $|\mathrm{Sr}|\left[\mathrm{Ga}_{2} \mathrm{Mg}_{2} \mathrm{~N}_{4}\right]$ [37] (see highlighted points in Fig. 2 for undoped ATN-ZnO and ATN-GaN). To investigate the stabilizing influence of doping we used DF calculations to probe the thermodynamic favorability of introducing dopants into TBSs and to assess the relative energies of different $\mathrm{K}$-Li-doped $\mathrm{ZnO}$ polymorphs (wurtzite, ATN and SOD). In all cases care was taken to find the lowest energy dopant positions. These calculations demonstrate that synthesis of ATN- $|\mathrm{K}|\left[\mathrm{Zn}_{3} \mathrm{LiO}_{4}\right]$ from the corresponding binary oxides (i.e., $\mathrm{ZnO}_{\text {wurtzite }}, \mathrm{K}_{2} \mathrm{O}, \mathrm{Li}_{2} \mathrm{O}$ ) is thermodynamically favored $\left(-15 \mathrm{~kJ} / \mathrm{mol}|\mathrm{K}|_{1 / 4}\left[\mathrm{Zn}_{3 / 4} \mathrm{Li}_{1 / 4} \mathrm{O}\right]\right)$ helping to rationalize the corresponding experimental synthesis. We also find that the hypothetical SOD- $|\mathrm{K}|\left[\mathrm{Zn}_{2} \mathrm{LiO}_{3}\right]$ structure is also similarly energetically favored $\left(-14 \mathrm{~kJ} / \mathrm{mol}|\mathrm{K}|_{1 / 3}\left[\mathrm{Zn}_{2 / 3} \mathrm{Li}_{1 / 3} \mathrm{O}\right]\right)$. Further, in both cases these polymorphs become (upon doping) more stable than the correspondingly doped wurtzite- $\mathrm{ZnO}$ structure (by $9 \mathrm{~kJ} / \mathrm{mol}|\mathrm{K}|_{1 / 4}\left[\mathrm{Zn}_{3 / 4} \mathrm{Li}_{1 / 4} \mathrm{O}\right]$ for doped ATN and by $5 \mathrm{~kJ} / \mathrm{mol}|\mathrm{K}|_{1 / 3}\left[\mathrm{Zn}_{2 / 3} \mathrm{Li}_{1 / 3} \mathrm{O}\right]$ for doped SOD). We note that with increasing doping, wurtzite- $\mathrm{ZnO}$ develops internal voids and/or layers indicative of the instability of doping this relatively dense structure. These calculations suggest that doping with pairs of charge-compensating cations, analogous to methods routinely used to prepare low-density silicate zeolites [15], may be a promising alternative to the synthesis of undoped phases using the low temperature deposition route as employed by Jansen et al. [10]. Nevertheless, based on their calculated low energy and structural stability, we reiterate that there is no compelling reason to suppose that many of our predicted undoped polymorphs cannot be synthesized directly.

In summary our detailed theoretical investigation shows that the paucity of low-density polymorphs observed for the majority of inorganic solids is very likely not due to significant energetic or structural constraints. Rather to the contrary, our wide ranging study suggests that the dense 


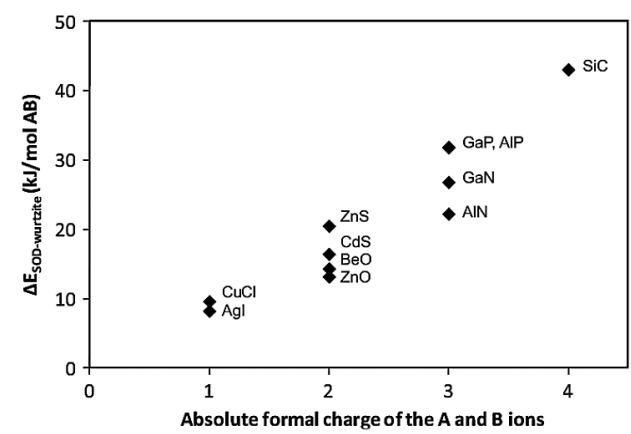

FIG. 3. Energy differences between the SOD and wurtzite polymorphs ( $\left.\Delta E_{\mathrm{SOD} \text {-wurtzite }}\right)$ for a range of TBSs plotted against the absolute formal charges of the constituent ions. To highlight the generality of the observed correlation, points for other TBSs are also included $(\mathrm{CuCl}, \mathrm{BeO}, \mathrm{AlN}, \mathrm{AlP})$.

spectrum of low-density polymorphs (experimentally observed for silica) is a general property of tetrahedrallycoordinated solids and perhaps all simple solids. We hope that our predictive study will inspire more experimental efforts into investigating low-density polymorphism in inorganic systems.

We acknowledge the Spanish Ministry of Science and Innovation for grant FIS2008-02238 and a Juan de la Cierva grant for M. A.Z. and support from Generalitat de Catalunya grant 2009SGR1041 and XRQTC. Computer time on MareNostrum (Barcelona Supercomputing Center) is also acknowledged. Dr. A. Berenguer, Dr. W. Sangthong, and Dr. A. A. Sokol are thanked for useful discussions.

*To whom all correspondence should be addressed.

[1] A. M. Guloy, R. Ramlau, Z. J. Tang, W. Schnelle, M. Baitinger, and Y. Grin, Nature (London) 443, 320 (2006).

[2] D. S. Wragg, R.E. Morris, and A.W. Burton, Chem. Mater. 20, 1561 (2008).

[3] M. M. J. Treacy, K. H. Randall, S. Rao, J. A. Perry, and D. J. Chadi, Z. Kristallogr. 212, 768 (1997).

[4] O. Delgado-Friedrichs, A. W. M. Dress, D. H. Huson, J. Klinowski, and A. L. Mackay, Nature (London) 400, 644 (1999).

[5] Y. Li, J. Yu, D. Liu, W. Yan, R. Xu, and Y. Xu, Chem. Mater. 15, 2780 (2003).

[6] M. M. J. Treacy, I. Rivin, E. Balkovsky, K. H. Randall, and M. D. Foster, Microporous Mesoporous Mater. 74, 121 (2004).

[7] D. J. Earl and M. W. Deem, Ind. Eng. Chem. Res. 45, 5449 (2006).

[8] M.D. Foster, A. Simperler, R. G. Bell, O. DelgadoFriedrichs, F. A. A. Paz, and J. Klinowski, Nature Mater. 3, 234 (2004).

[9] M. A. Zwijnenburg and R. G. Bell, Chem. Mater. 20, 3008 (2008).

[10] Y. Liebol-Ribeiro, D. Fischer, and M. Jansen, Angew. Chem., Int. Ed. 47, 4428 (2008).
[11] Z.P. Cancarevic, J. C. Schön, and M. Jansen, Chem. Asian J. 3, 561 (2008).

[12] G. S. Oleinik and N. V. Danilenko, Russian Chemical Reviews 66, 553 (1997).

[13] Group IV Elements, IV-IV and III-V Compounds, edited by O. Madelung, U. Rössler, and M. Schulz, LandoltBörnstein, Group III Vol. 41A (Springer-Verlag, Berlin, 1999).

[14] II-VI and I-VII Compounds, Semimagnetic Compounds, edited by O. Madelung, U. Rössler, and M. Schulz, Landolt-Börnstein, Group III Vol. 41B (Springer-Verlag, Berlin, 1999).

[15] Ch. Baerlocher, L. B. McCusker, and D. H. Olson, Atlas of Zeolite Framework Types (Elsevier, Amsterdam, 2007), 6th ed. (revised).

[16] D. K. Smith, C.F. Cline, and S. B. Austerman, Acta Crystallogr. 18, 393 (1965).

[17] J. Wang, A. J. Kulkarni, K. Sarasamak, S. Limpijumnong, F. J. Ke, and M. Zhou, Phys. Rev. B 76, 172103 (2007).

[18] B. Morgan, Phys. Rev. B, 80, 174105 (2009).

[19] S. Hamad and C. R. A. Catlow, J. Cryst. Growth, 294, 2 (2006).

[20] J. Carrasco, F. Illas, and S. T. Bromley, Phys. Rev. Lett. 99, 235502 (2007).

[21] M. B. Watkins, S. A. Shevlin, A. A. Sokol, B. Slater, C. R. A. Catlow, and S. M. Woodley, Phys. Chem. Chem. Phys. 11, 3186 (2009).

[22] K. Doll, J.C. Schön, and M. Jansen, Phys. Rev. B 78, 144110 (2008).

[23] J. D. Gale, Z. Kristallogr. 220, 552 (2005).

[24] L. Whitmore, A. A. Sokol, and C. R. A. Catlow, Surf. Sci. 498, 135 (2002).

[25] J. Perdew, K. Burke, and Y. Wang, Phys. Rev. B 54, 16533 (1996).

[26] P.E. Blöchl, Phys. Rev. B 50, 17953 (1994).

[27] G. Kresse and D. Joubert, Phys. Rev. B 59, 1758 (1999).

[28] R. A. Robie and B. S. Hemingway, US Geological Survey Bulletin (United States Government Printing Office, Washington, 1995), Vol. 2131.

[29] S. J. Deore and A. Navrotsky, Am. Mineral. 91, 400 (2006).

[30] C. Y. Yeh, Z. W. Lu, S. Froyen, and A. Zunger, Phys. Rev. B 46, 10086 (1992).

[31] K. Karch, F. Bechstedt, F. Pavone, and D. Strauch, Phys. Rev. B 53, 13400 (1996).

[32] C.R. A. Catlow, S. A. French, A. A. Sokol, A. A. AlSundaidi, and S. M. Woodley, J. Comput. Chem. 29, 2234 (2008).

[33] F. Decremps, J. Pellicer-Porres, F. Datchi, J.P. Itie, A. Polian, F. Baudelet, and J.Z. Jiang, Appl. Phys. Lett. 81, 4820 (2002).

[34] J. Lin, E. Cates, and P. Bianconi, J. Am. Chem. Soc. 116, 4738 (1994).

[35] F. Xu, Y. Xie, S. Zhang, and L. Shi, New Journal of Chemistry 27, 565 (2003).

[36] R. Hoppe and E. Siepp, J. Solid State Chem. 72, 52 (1988).

[37] D. G. Park, Y. Dong, and F. J. DiSalvo, Solid State Sci. 10, 1846 (2008).

[38] http://www.hypotheticalzeolites.net 